\title{
Free thyroxine in needle washout after fine needle aspiration biopsy of toxic thyroid nodules
}

Nikolai Raikov, Boyan Nonchev², Borislav Chaushev³, Diyana Vjagova4, Svetoslav Todorov4, Yana Bocheva5, Daniela Malceva ${ }^{4}$, Snejinka Vicheva ${ }^{6}$, Asyia Raikova $^{7}$, Antoaneta Argatska ${ }^{2}$, Miroslav Raikov ${ }^{8}$

'Medical Complex "Plus", Varna, Bulgaria

${ }^{2}$ Clinic of Endocrinology, Medical University, Plovdiv, Bulgaria

3Department of Nuclear Medicine "Saint Marina", Varna, Bulgaria

${ }^{4}$ Clinical Laboratory "Status", Varna, Bulgaria

${ }^{5}$ Central Clinical Laboratory "Saint Marina", Varna, Bulgaria

"Department of clinical pathology, Oncological Dispensary "Marko Markov", Varna, Bulgaria

'Department of Internal Medicine, MBAL Dobrich, Bulgaria

${ }^{8}$ Medical University, Varna, Bulgaria

[Received 24 VI 2015; Accepted 26 X 2015]

\begin{abstract}
The main diagnostic tool for toxic adenomas (TA) is radionuclide imaging indicated in patients with evidence of thyroid nodules in combination with thyrotoxic syndrome. Thyroid ultrasound and fine-needle aspiration biopsy (FNAB) are widely used for the evaluation of thyroid masses. There is no literature data concerning the utility of FNAB and related tests for the diagnosis of hyperfunctioning thyroid nodules. The purpose of this study is to determine the levels of free thyroxine (FT4) in the needle washout after FNAB of hot thyroid nodules. The results of our study show that the FT4 levels in needle washout from TA were significantly higher than the surrounding parenchyma and correlated with the hormonal changes in patients with thyroid hyperfunctioning nodules. Further studies on a large number of patients are needed to refine the diagnostic value of this method and evaluate its importance in quantitative risk assessment of thyroid autonomy.
\end{abstract}

KEY words: fine-needle aspiration biopsy (FNAB), hot thyroid nodules - toxic adenoma (TA), thyroid radionuclide imaging

Nuclear Med Rev 2016; 19, 1: 18-21

\section{Background}

Thyroid autonomy is a common finding in iodine-deficient areas where it accounts for $60 \%$ of the cases with thyrotoxicosis (toxic adenoma - 10\%; toxic multinodular goiter $-50 \%$ ) [1, 2]. The main diagnostic tool to ascertain toxic adenomas (TA) is radionuclide imaging which is indicated in patients with clinical or ultrasound (US) evidence of thyroid nodules in combination with thyrotoxic syndrome. TA are most commonly diagnosed due to clinically overt thyrotoxicosis [3]. Thyroid ultrasound and fine-needle aspiration biopsy (FNAB) are widely used for the evaluation of thyroid masses [4]. There is no literature data on the utility of FNAB and related tests for the diagnosis of hyperfunctioning thyroid nodules.

Correspondence to: Borislav Chaushev, MD PhD

Specialist of Nuclear Medicine

Department of Nuclear Medicine and Metabolic therapy

MBAL "St. Marina", Varna, Bulgaria

Varna 9010, $1 \mathrm{Hr}$. Smirzenski Str.

E-mail: bchaushev@gmail.com

\section{Aim}

To determine the levels of free thyroxine (FT4) in the needle washout after FNAB of hot thyroid nodules.

\section{Material and methods}

Nineteen patients (mean age $51.79 \pm 2.97$; from 29 to 71 years) were included in the study. Male/Female ratio was 1: 3.75 (Table 1). All patients had hormonal evidence of thyrotoxicosis (mainly subclinical) and hyperfunctioning nodules detected on thyroid radionuclide scan. Outpatients were referred to an endocrinologist for thyroid gland assessment due to the presence of palpable thyroid mass or changes in thyroid hormones levels. Medical history at the time of primary evaluation did not reveal concomitant autoimmune thyroid diseases, previous treatment with antithyroid drugs, levothyroxine, iodine supplements or radioiodine treatment. According to the radionuclide imaging findings, nodules were classified as decompensated (hyperfunctioning nodules with suppression of extranodular gland), subcompensated (increased uptake in the nodule with slight suppression of extranodular parenchyma) and compensated (nodules with increased uptake compared with adjacent tissue without 
Table 1. Characteristics of the studied patients

\begin{tabular}{|c|c|c|}
\hline Patients & $\mathrm{n}$ & $\%$ \\
\hline \multicolumn{3}{|l|}{ Gender } \\
\hline Female & 15 & 78.95 \\
\hline Male & 4 & 21.05 \\
\hline \multicolumn{3}{|l|}{ Age (years) } \\
\hline Mean & \multicolumn{2}{|c|}{$51.79 \pm 2.97$} \\
\hline Range & \multicolumn{2}{|c|}{$29-71$} \\
\hline \multicolumn{3}{|l|}{ Serum levels of: } \\
\hline TSH & \multicolumn{2}{|c|}{$0.16 \pm 0.03$} \\
\hline FT4 & \multicolumn{2}{|c|}{$19.81 \pm 1.28$} \\
\hline FT3 & \multicolumn{2}{|c|}{$6.57 \pm 0.37$} \\
\hline Volume of the nodule [ml] & \multicolumn{2}{|c|}{$2.70 \pm 0.39$} \\
\hline Volume of the thyroid gland [ml] & \multicolumn{2}{|c|}{$13.56 \pm 0.61$} \\
\hline \multicolumn{3}{|l|}{ Radionuclide scan } \\
\hline Compensated & 1 & 5.26 \\
\hline Subcompensated & 13 & 68.42 \\
\hline Decompensated & 5 & 26.32 \\
\hline
\end{tabular}

suppression of extranodular thyroid tissue). Ultrasound (US) examination of the neck region was performed by one investigator using linear transducer with modulated 10-18 MHz frequency - LA435 (Esaote MyLab 25 Gold). US guided FNAB of the described nodular formations was conducted and a sample for cytological examination was prepared. The needle was washed out after the dispersal of the punctate onto a slide with $1 \mathrm{ml}$ saline and levels of free thyroxine (FT4) in the washout were measured by electro-chemiluminescence immunoassay (ECLIA) (Roche Elecsys 2010 analyzer). An additional washout sample from the surrounding parenchyma was prepared according to the method described above. Serum levels of thyroid stimulating hormone TSH (RR 0.27-4.20 $\mu \mathrm{lU} / \mathrm{ml})$; free thyroxine FT4 (RR 10-25 pmol/l); free triiodothyronine FT3 (RR 2.8-7.1 pmol/l) were also determined as blood samples obtained before FNAB. Thyroid scintigraphy was performed using ${ }^{99 \mathrm{~m} T c-p e r t e c h n e t a t e ~} 2-5 \mathrm{mCi}$, given as an intravenous injection. Patient preparation included exclusion of medications that are known to affect thyroid uptake. Thyroid scan was performed as a planar scan on a gamma camera ADAX Vertex with low energy general purpose collimator, planar acquisition, $512 \times 512$ matrix for 5 minutes or 300000 counts. Patients with preexisting autoimmune thyroid disorders were excluded from the study. All patients signed an informed consent form to participate in the study. For statistical analysis exact Fisher test and correlation analysis were applied.

\section{Results}

The results from cytological examination of the specimen from all patients demonstrated benign thyroid nodules (Figure 1).

The analysis of the data showed significantly higher levels of FT4 in the washout from the nodules compared with those from the surrounding thyroid tissue (Figure 2).

Considering that hormonal changes in patients with toxic thyroid nodules result from excessive hormonal production from the adenoma, a correlation between the levels of FT4 in the washout samples and serum levels of thyroid hormones and TSH was presumed. The analysis found statistically significant correla-

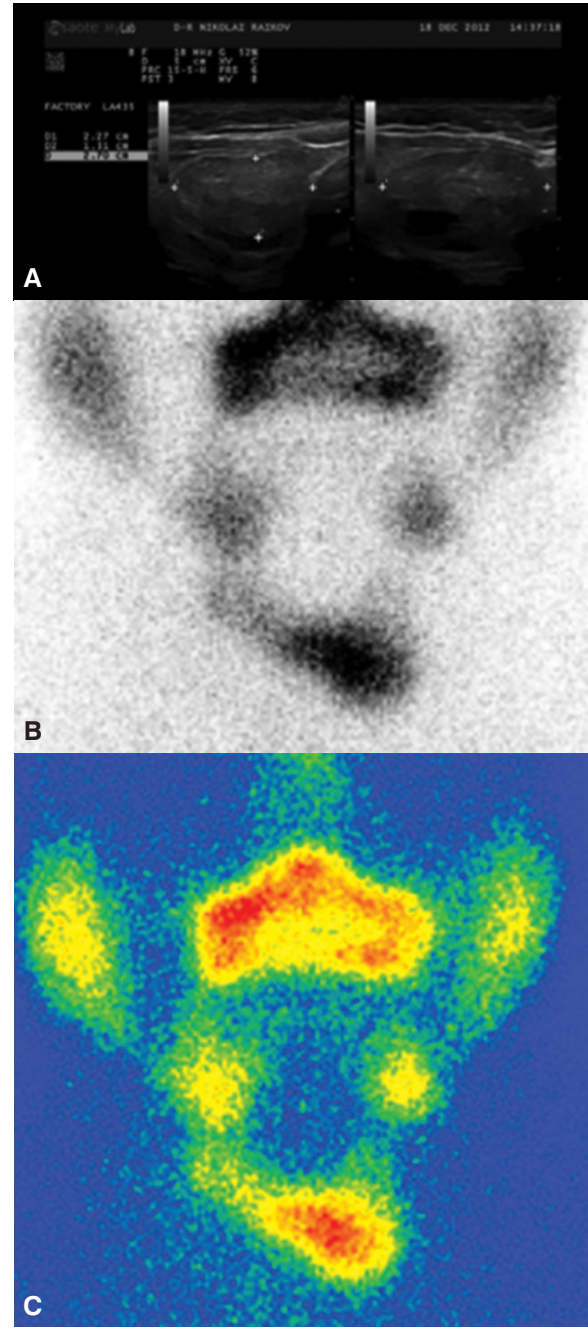

Figure 1. A case of 52-year-old female presenting with hot thyroid nodule on radionuclide scan $\mathbf{B}$ and $\mathbf{C}$. US features: a dominant solid isoechoic with peripheral and intranodular vascularization nodule sized 23/14/27MM: A. FT4 needle wash-out from the nodule $=137 \mathrm{pmol} / \mathrm{l}$. FT4 needle washout from the surrounding parenchyma $=1.48 \mathrm{pmol} / \mathrm{l}$. Cytological result: Bethesda category 2 (benign lesion)

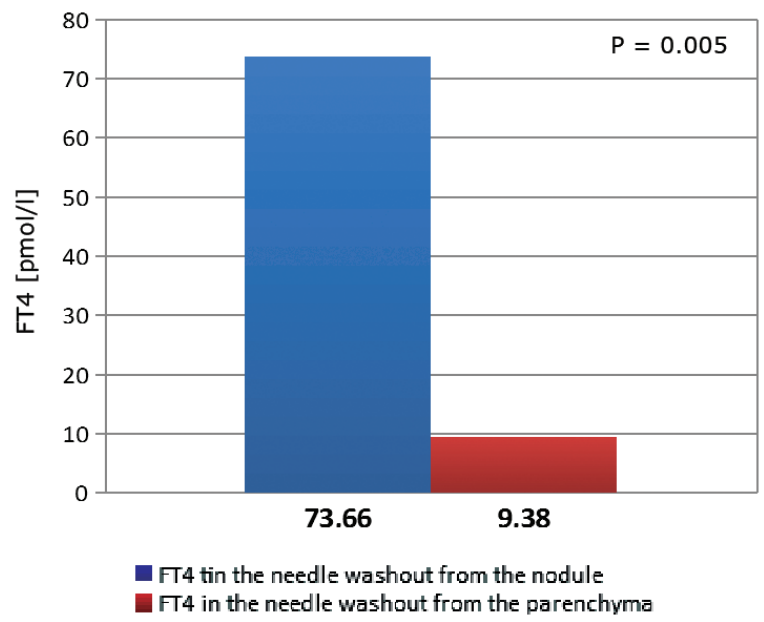

Figure 2. Mean FT4 in the needle washout from the nodule compared to surrounding parenchyma 


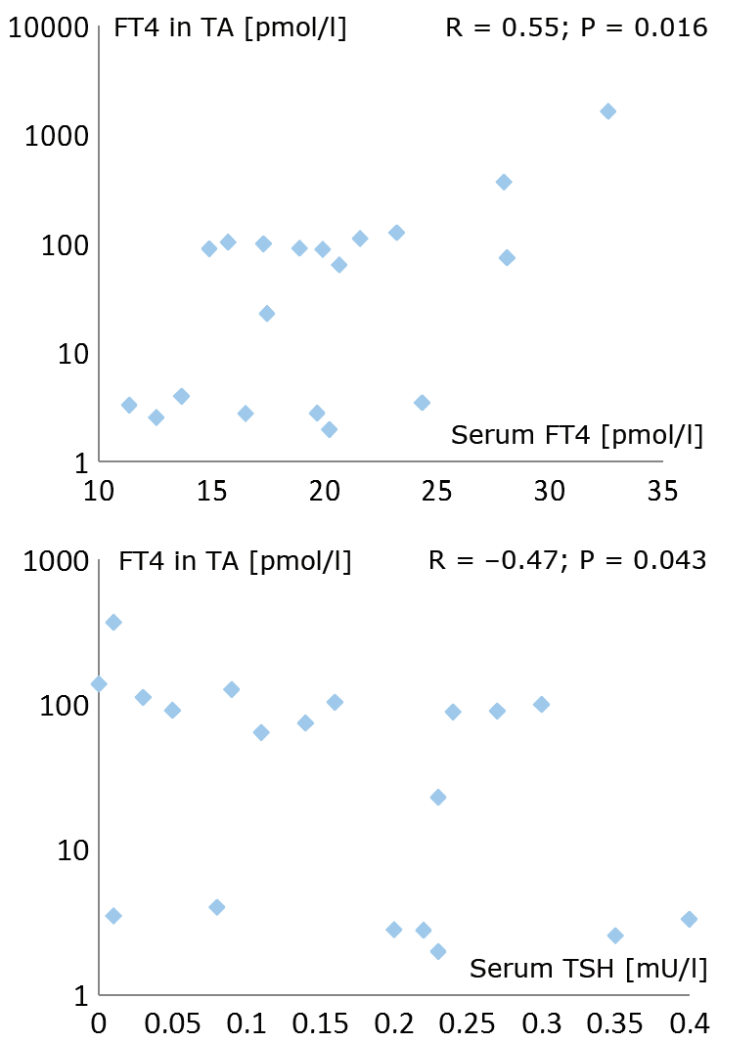

Figure 3. Correlation between FT4 needle washout form TA and serum TSH and FT4

tion between FT4 in the washout and serum FT4 and a negative correlation with the TSH concentrations (Figure 3). No correlation with serum levels of FT3 was established.

Correlation between the levels of FT4 in washout from thyroid parenchyma and serum FT4 $(r=0.53, P=0.021)$ and FT3 $(r=0.47$; $P=0.042$ ) was found.

The mean values of FT4 in the washout from the decompensated TA were significantly higher (Table 2).

It is known that the severity of hormonal changes in patients with TA is related to the size of the TA [5]. In the present study a similar correlation between the nodule volume and serum TSH, FT3 and FT4 was found. However, a correlation between the levels of FT4 in fine needle washout and the volume of the TA and the thyroid volume was not ascertained.

\section{Discussion}

Currently, the diagnosis of toxic adenoma is based on three main criteria: clinical and/or hormonal evidence of thyrotoxicosis, palpable or ultrasonographic finding of thyroid mass and increased radionuclide uptake in the suspected nodule along with suppressed activity in the remainder of the thyroid [3]. FNAB is not a routine diagnostic method of toxic adenomas due to the low risk of malignancy, but provides valuable information on the benign nature of the formations especially in the absence of radionuclide imaging at the time of evaluation. Long-term studies of patients with TA have shown that the natural course of the disease is slow and requires years or decades to develop thyrotoxic syndrome. In a study of Sandrock et al. authors reported $4.1 \%$ annual incidence of thyrotoxicosis in a group of 375 untreated euthyroid patients with TA [6].

Measurement of bioactive substances in needle washout after FNAB is widely used in clinical thyroidology. At present, the most extensive research has been made on the accuracy of thyroglobulin in needle washout which has shown the highest sensitivity and specificity in diagnosing of metastatic lymph nodes in patients with differentiated thyroid carcinoma [7]. There are also data regarding the diagnostic value of parathyroid hormone in patients with adenomas of the parathyroid glands [8] and calcitonin in medullary carcinomas [9]. The review of the literature does not provide studies focused on measurement of hormonal parameters from the needle washout after FNAB of hyperfunctioning thyroid nodules.

In the current study we analyzed the data of 19 patients with TA in whom the FT4 level from needle washout is assessed. The samples were prepared following the methodology applied for thyroglobulin measurement in fine needle washout from metastatic lymph nodes [10]. The results showed significantly higher levels of FT4 in washout from the nodules compared with the surrounding thyroid parenchyma. These findings are consistent with the evidence of focal hormone oversecretion from the adenoma and the relative suppression of the intact thyroid tissue. The established correlation between FT4 in washout from TA and serum levels of FT4 and the negative correlation with TSH confirms the relation between TA and thyroid function tests and can provide useful data on the activity of overactive thyroid tissue. Hot nodules do not have the same functional activity and if the serum hormone levels are directly related to the volume of the nodule, the hormonal production from a similar volume is different. We did not establish a correlation between the thyroid adenoma volume and the level of FT4 in the washout which is in accordance with this standpoint. Despite the limited number of cases at this phase of the study, significantly higher mean levels of FT4 in decompensated nodules were found compared with subcompensated and compensated adenomas. It can be assumed that measurement of FT4 in washout from TA can provide circumstantial evidence of nodule hyperfunction and justify further tests to verify the diagnosis. It is stated in the current guidelines that the clinical approach to the diagnosis and treatment of thyroid nodules should be determined by the results of ultrasound examination in combination with the cytological result after FNAB [4]. The measurement of the FT4 in needle washout after FNAB provides additional information to the diagnostic approach.

Table 2. FT4 needle washout form TA and from surrounding parenchyma in compensated and decompensated nodules

$\begin{array}{lcc} & \text { Compensated/subcompensated } & \text { decompensated } \\ \text { FT4 in needle washout from TA } & 40.62 \pm 13.29 & 145.25 \pm 45.62 \\ \text { FT4 in needle washout from parenchyma } & 5.76 \pm 4.08 & 17.21 \pm 12.63 \\ \text { Mean FT4 in nodule/FT4 in parenchyma ratio } & 37.58 & 61.18\end{array}$




\section{Conclusion}

The results of our study show that the FT4 levels in needle wash-out from TA were significantly higher than the surrounding parenchyma and correlated with the hormonal changes in patients with thyroid hyperfunctioning nodules. Further studies on a large number of patients are needed to refine the diagnostic value of this method. Based on our preliminary data, we consider FT4 in washout from TA a potential diagnostic tool for the early diagnosis of toxic thyroid adenoma.

\section{References}

1. Laurberg P, Pedersen KM, Vestergaard $\mathrm{H}$ et al. High incidence of multinodular toxic goitre in the elderly population in a low iodine intake area vs. high incidence of Graves' disease in the young in a high iodine intake area: comparative surveys of thyrotoxicosis epidemiology in East-Jutland Denmark and Iceland. J Intern Med 1991; 229: 415-420.

2. Delange $F$, de Benoist $B$, Pretell $E$ et al. lodine deficiency in the world: where do we stand at the turn of the century? Thyroid 2001; 11: 437-447.

3. Paschke R. Toxic adenoma and toxic multinodular goiter. Chapter 20. In: The Thyroid - a fundamental and clinical text. Thenth edition. Werner \& Ingbar's 2012: 400-409.
4. Gharib H, Papini E, Paschke R et al. AACE/AME/ETA Task Force on Thyroid Nodules. American Association of Clinical Endocrinologists, Associazione Medici Endocrinologi, and European Thyroid Association Medical guidelines for clinical practice for the diagnosis and management of thyroid nodules: executive summary of recommendations. Endocr Pract 2010; 16: $468-475$.

5. Homburger JL. Evolution of toxicity in solitary nontoxic autonomously functioning thyroid nodules. J Clin Endocrinol MetDb 1980; 50: 1089.

6. Sandrock D, Olbricht T, Emrich D et al. Long-term follow-up in patients with autonomous thyroid adenoma. Acta Endocrinol (Copenh) 1993; 128: 51.

7. Grani G, Fumarola A. Thyroglobulin in lymph node fine-needle aspiration washout: a systematic review and meta-analysis of diagnostic accuracy. J Clin Endocrinol Metab 2014; 99: 1970-1982.

8. Triggiani V, Resta F, Giagulli VA et al. Parathyroid hormone determination in ultrasound-guided fine needle aspirates allows the differentiation between thyroid and parathyroid lesions: our experience and review of the literature. Endocr Metab Immune Disord Drug Targets 2013; 13: 351-358.

9. Trimboli P, Cremonini N, Ceriani L et al. Calcitonin measurement in aspiration needle washout fluids has higher sensitivity than cytology in detecting medullary thyroid cancer: a retrospective multicentre study. Clin Endocrinol (Oxf) 2014; 80: 135-140.

10. Zanella AB, Meyer EL, Balzan L et al. Thyroglobulin measurements in washout of fine needle aspirates in cervical lymph nodes for detection of papillary thyroid cancer metastases. Arq Bras Endocrinol Metabol 2010; 54: 550-554. 